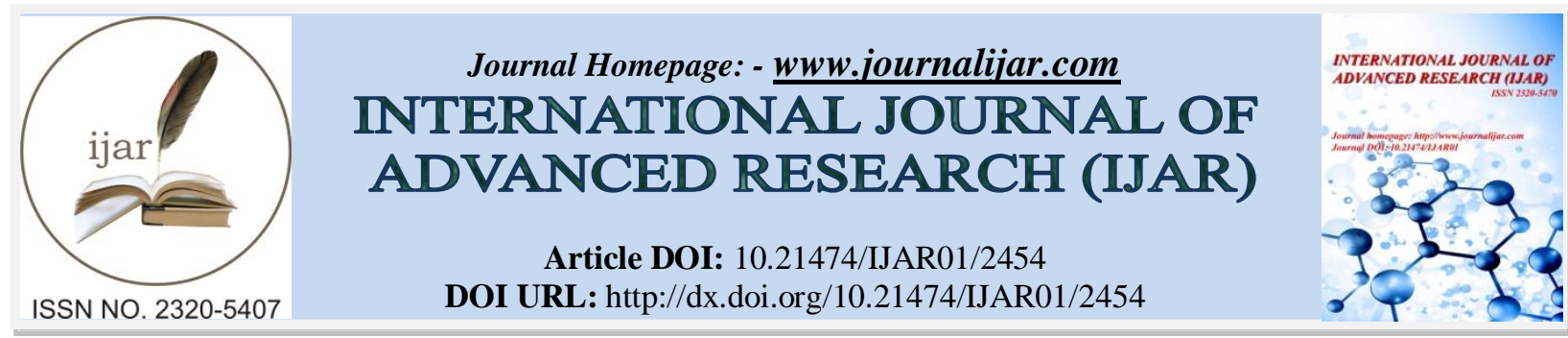

RESEARCH ARTICLE

\title{
MONOSTOTIC AND POLYOSTOTIC FORMS OF FIBROUS DYSPLASIA OF THE JAWS - CASE REPORT
}

\section{Dr Kumar Chandan Srivastava, Dr Nelson Aruldoss, Dr. Mansur Sathick, Dr. Deepti Shrivastava and Dr. Ravi David Austin.}

\section{Manuscript Info \\ Manuscript History \\ Received: 20 October 2016 \\ Final Accepted: 22 November 2016 \\ Published: December 2016}

Key words:-

Craniofacial Fibrous dysplasia

Monostotic form Polyostotic form.

\section{Abstract}

Fibrous Dysplasia is a developmental dysplastic disorder of bone in which the normal bone matrix is replaced by fibroblastic proliferation. Von Rechlinghausen was the first author who described it in 1891 and Lichtenstein was the person who introduced the term fibrous dysplasia. It can be monostotic $(70 \%)$ or polyostotic $(30 \%)$. The craniofacial localization occurs in $10 \%-25 \%$ of cases in monostotic forms and in $50 \%$ of cases in polyostotic forms. Almost all patients with extensive polyostotic forms of the disease have skull involvement, while most patients with craniofacial bone involvement have the monostotic form of the disease. Fibrous dysplasia essentially affects children and young adults, with no sex preference. Diagnosis is not always straightforward because the functional symptomology is often absent or not specific. Only symptomatic and/or gross forms are considered for treatment. We report two cases of fibrous dysplasia, one monostotic form involving only the maxilla and other polyostotic involving bilateral facial and skull bones.

Copy Right, IJAR, 2016,. All rights reserved.

\section{Introduction:-}

Fibrous Dysplasia is a developmental dysplastic disorder of bone in which the normal bone matrix is replaced by fibroblastic proliferation. It is a benign intramedullary fibro-osseous lesion originally described by Lichtenstein and Jaffe in 1938. The true incidence and prevalence of fibrous dysplasia is difficult to estimate accurately, but the lesions are not rare; they are reported to represent approximately 5\% to 7\% of benign bone tumors. Male to female ratio is equal. It is most frequently found in metaphysodiaphyseal region of long bones i.e. in rib (28\%), followed by femur $(23 \%)$, tibia and craniofacial bone (10-25\%). Sarcomatous transformation is rare. ${ }^{(1)}$ Some people with fibrous dysplasia have only one bone involved (monostotic), whereas other people have more than one bone involved (polyostotic). The disease may occur alone, or as part of a condition known as the McCune-Albright syndrome. McCune-Albright syndrome is characterized by fibrous dysplasia and other symptoms such as patches of pigmented skin (light brown or "café-au-lait" spots) and endocrine problems such as precocious puberty, hyperthyroidism, gigantism or acromegaly, Cushing's syndrome, and other rare conditions. 50\%-100\% of patient with polyostotic disease \& $10 \%$ patient with monostotic disease have craniofacial involvement. ${ }^{(2)}$ 


\section{Case Reports:- \\ Case Report 1:-}

A 25 year old female, complains of painless, slow growing swelling in the left posterior palatal region since 5 years. Recently, since 4 months, it became symptomatic with an onset of intermittent and moderate grade of pain. Recently it got associated with nasal obstruction and slight discharge from the left eye. Examination revealed a hard, non tender, smooth surfaced, diffuse swelling extending superiorly to the left infra-orbital margin and inferiorly $4 \mathrm{~cm}$ above the angle of the mouth. Medially, it extended up to the midline of nasal bridge and laterally up to the zygomatic prominence. Intraoral examination revealed buccal cortical expansion in the same region, which was later confirmed on maxillary occlusal view and computed tomography (CT) scan slices. Deciduous $1^{\text {st }}$ and $2^{\text {nd }}$ molar were retained with slight mobility along with the buccal displacement of the maxillary canine.

Intraoral periapical view showed an ill defined, homogenous radiopaque lesion involving the left posterior maxilla, obscuring the lamina dura of the adjacent teeth (Figure 1). CT scan showed homogenous, hyperdense lesion completely filling the left maxillary sinus with completely eroded buccal cortical plate and infiltrating into the nasal cavity compressing the inferior nasal conchae of the same side (Figure $2,3 \& 4$ ). On correlating clinical \& radiological features, a clinical diagnosis of Monostotic fibrous dysplasia was made, which later got confirmed with histopathological examination. (Figure 5)

\section{Case Report 2;-}

A 17 year old female complains of painless, gradually progressive swelling in her right side of the face since 5 years. It was not associated with nasal obstruction, or discharge from eye or nose. Gross facial asymmetry was observed due to a non tender, bony hard, diffuse swelling in the right middle third region of the face. It extended supero-inferiorly from $1 \mathrm{~cm}$ below the infra-orbital margin to the angle of the mouth and antero-posteriorly from right lateral border of the nose, obliterating the nasolabial fold to $3 \mathrm{~cm}$ short off tragus. On intra oral examination, obliteration of both right and left upper buccal vestibule was evident due to a non tender, bony hard, diffuse, smooth surface swelling causing buccal cortical expansion from canine region to maxillary tuberosity with the maximum dimension at the molar region. CT scan of craniofacial region showed bilateral, homogenous hyper dense lesion (Figure 7) involving multiple facial bones viz nasal, lacrimal, maxillary, zygomatic and inferior nasal conchae (Figure 8). Among cranial bones, frontal, ethamoid, sphenoid, squamous parts of occipital and temporal were involved (Figure 9). Blood and Biochemical investigation showed Serum Calcium (9.3 mg/dl) and phosphorus (4.1 $\mathrm{mg} / \mathrm{dl}$ ) within normal range whereas alkaline phosphatase (240 IU/L) turned out to be raised. Considering clinical, radiological, serum chemistry and histopathological report, diagnosis of Polyostotic fibrous dysplasia of craniofacial type was confirmed.

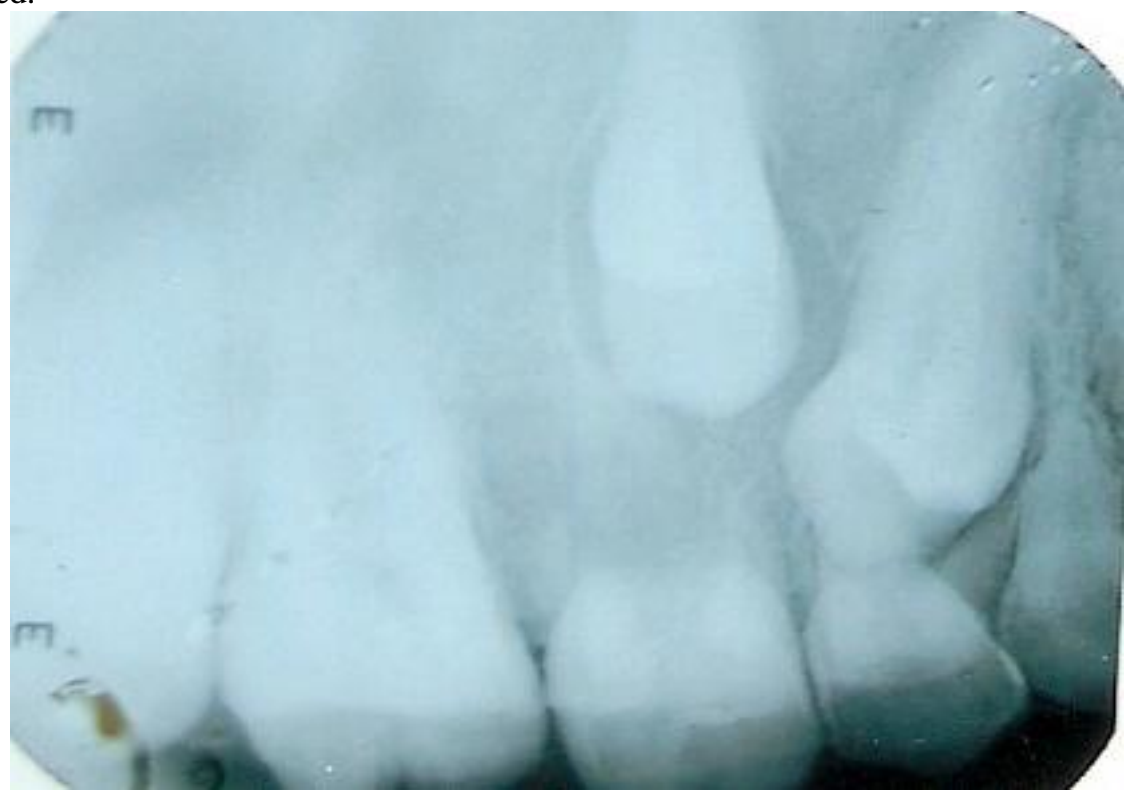

Figure 1:- Intraoral periapical view showing diffuse homogenous radiopacity in left maxillary posterior giving ground glass appearance. 


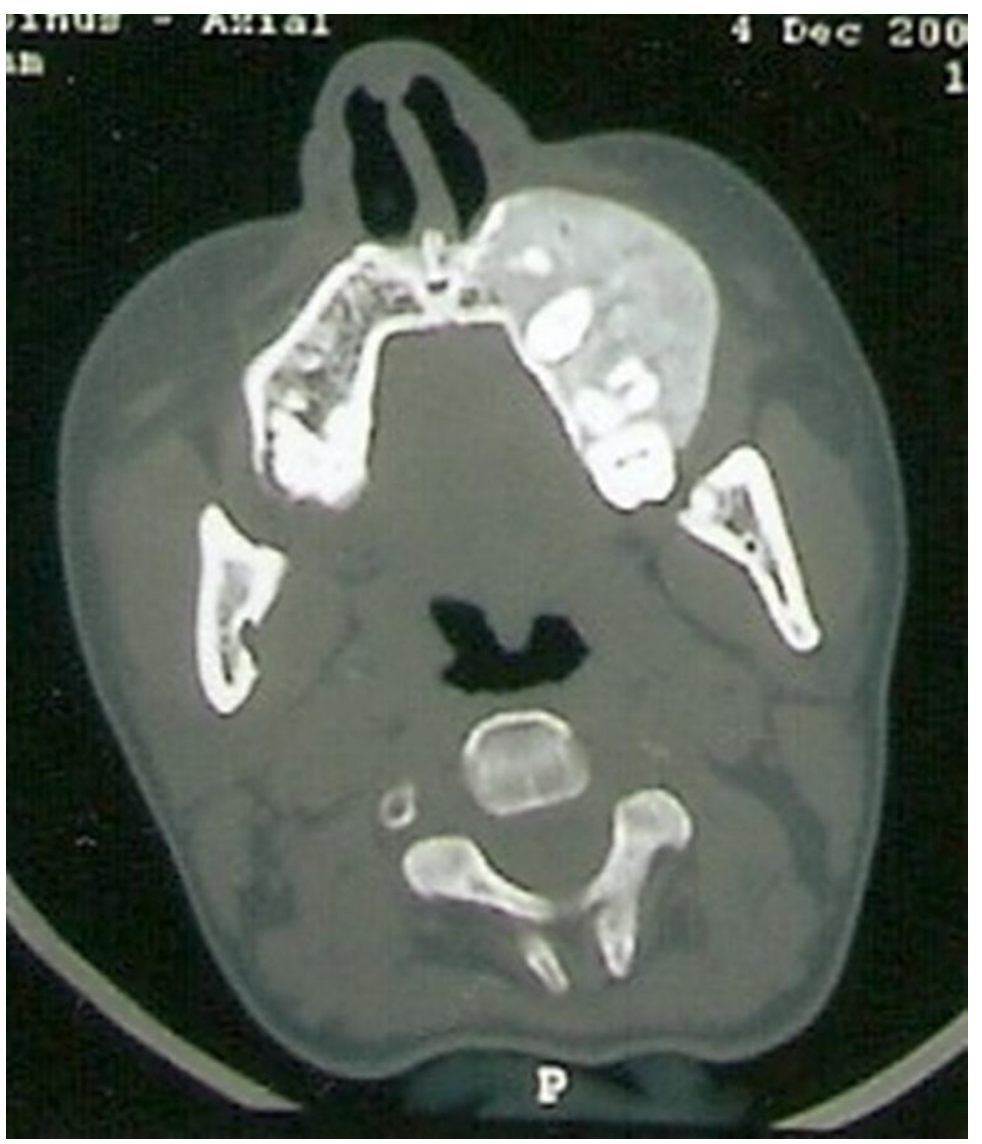

Figure 2:- $2.5 \mathrm{~mm}$ axial CT imaging was obtained through the facial bones and bone window is utilized for this study. Image shows unilateral (left) expansion of the maxillary arch with resorbed buccal cortical plate.

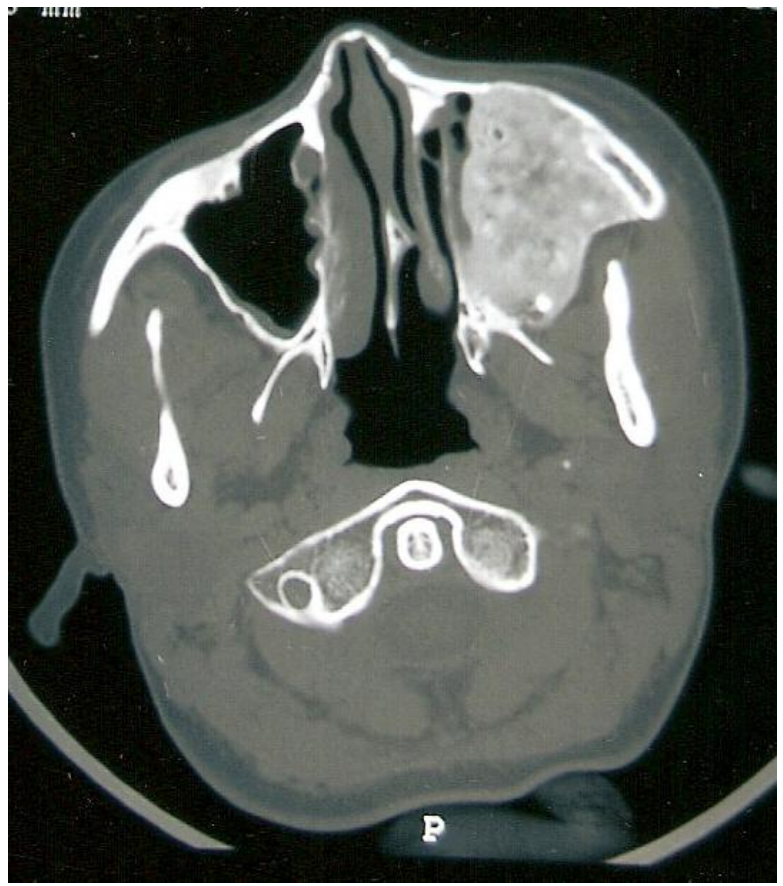

Figure 3:- Axial CT image showing radioopaque lesion completely filling / opacification of left maxillary sinus with thinning of buccal cortical plate. 


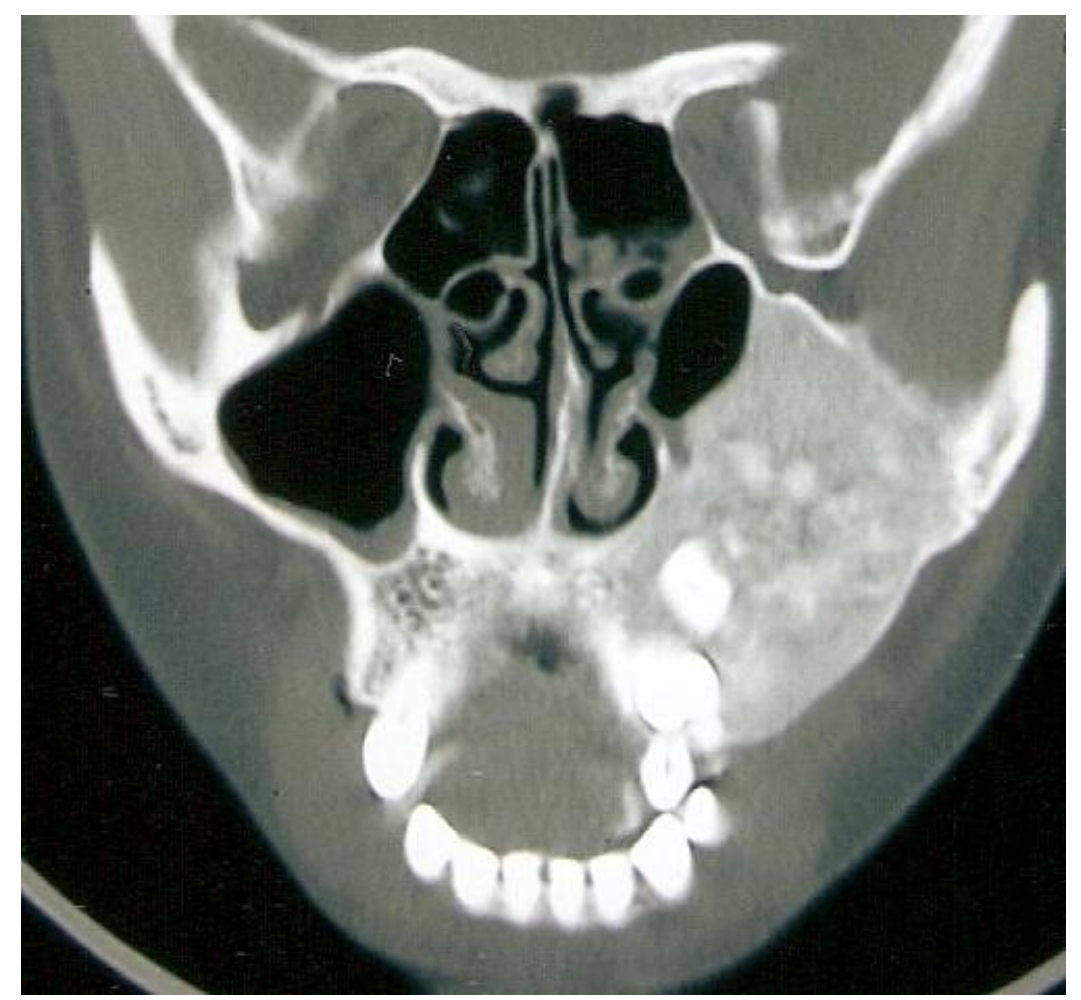

Figure 4:- Coronal CT image showing homogenous radiopaque lesion involving maxillary sinus with erosion of medial wall leading to encroachment into the inferior nasal conchae medially and maxillary bone inferiorly.

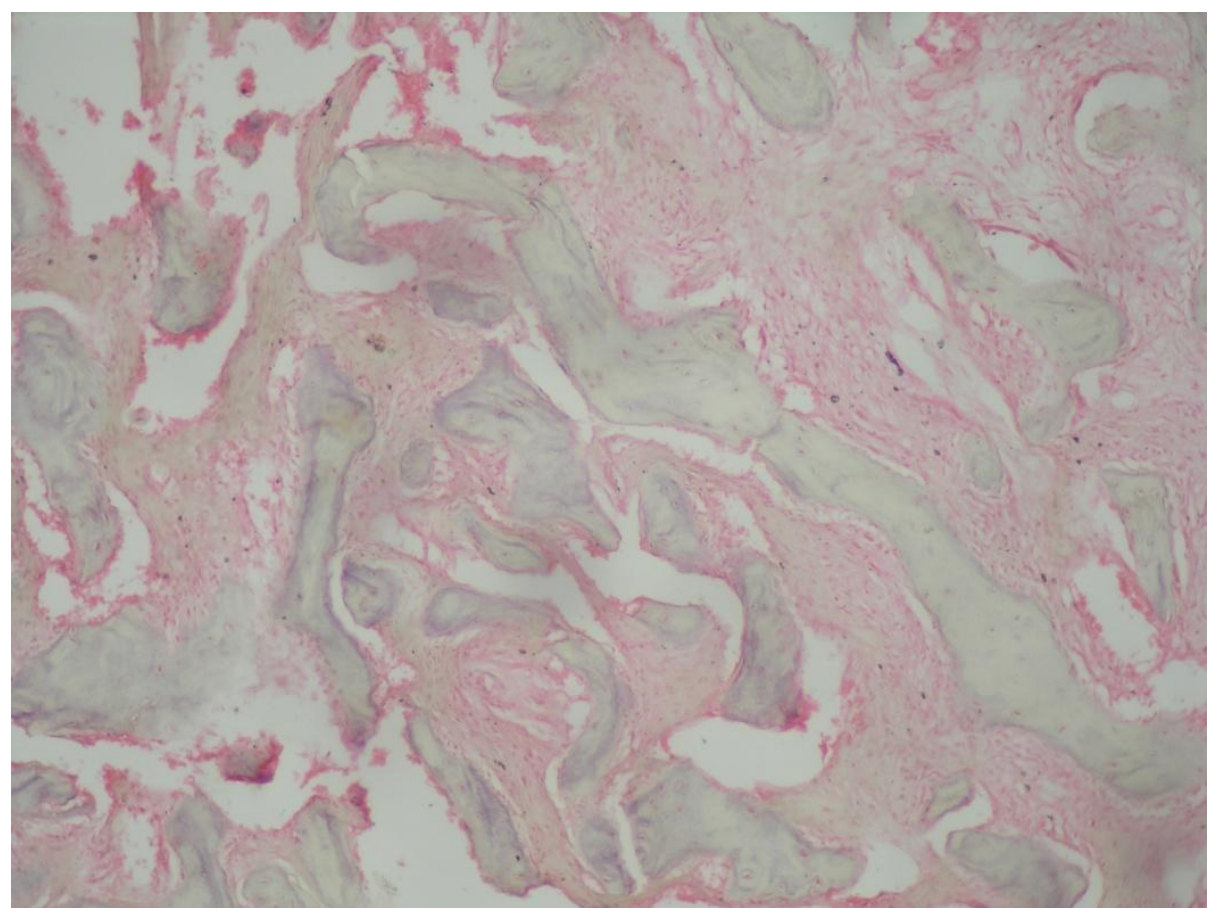

Figure 5:- A photomicrograph showing "C" or "Chinese letter" shaped bony trabeculae in a highly cellular connective tissue (H/E, 10X). 


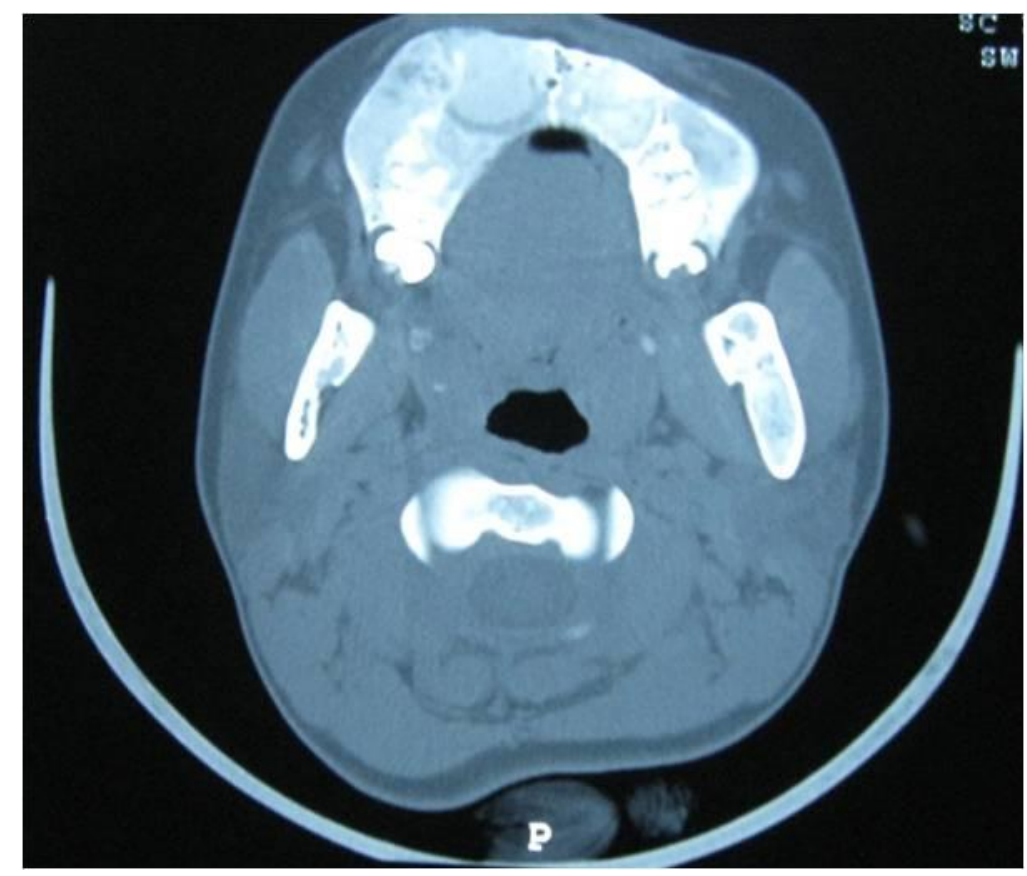

Figure 6:- Axial CT image showing bilateral expansion of the maxillary arch with resorbed buccal cortical plate.

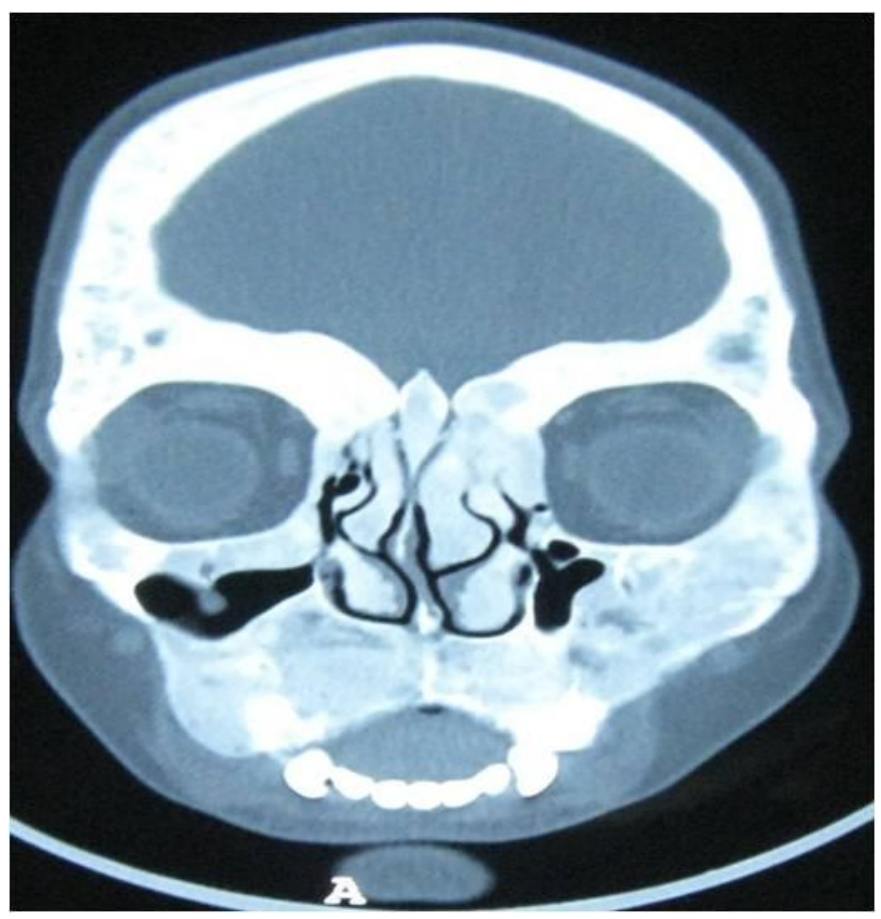

Figure 7:- Coronal CT image showing expansion of frontal, ethamoid, sphenoid, maxillary, nasal, lacrimal, zygomatic and inferior nasal conchae bones. 


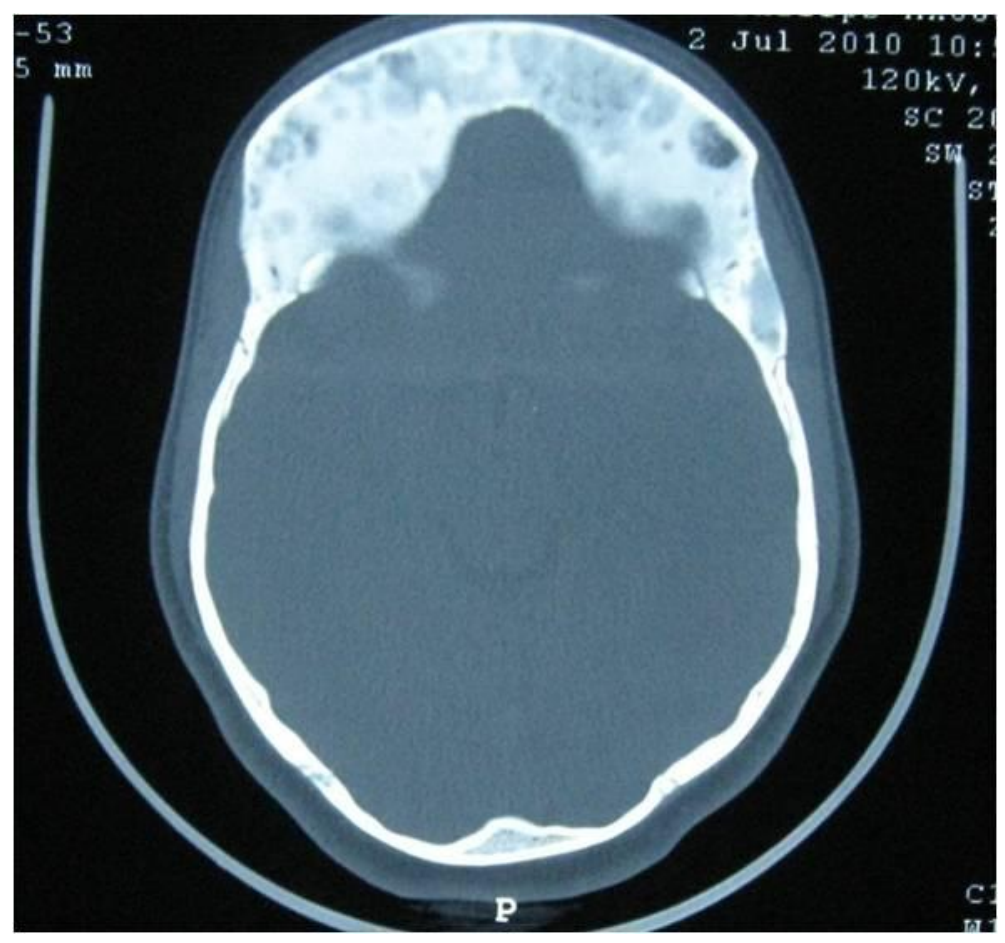

Figure 8:- Axial CT image showing expansion of the frontal and temporal bones.

\section{Discussion:-}

The etiology of fibrous dysplasia has been linked with a mutation in the Gs $\alpha$ gene that occurs after fertilization in somatic cells and is located at chromosome 20q13.2-13.3. ${ }^{(3)}$ Fibrous dysplasia is postulated to occur as a result of a developmental failure in the remodeling of primitive bone to mature lamellar bone and a failure of the bone to realign in response to mechanical stress. Failure of maturation leaves a mass of immature isolated trabeculae enmeshed in dysplastic fibrous tissue that are turning over constantly but never completing the remodeling process. (4)

The clinical presentation depends on the site, duration, extent and nature of the lesion, which can affect any bone in the body. It ranges from a mild local swelling with little or no pain to a gross deformity with complications such as proptosis, visual disturbance and sensorineural hearing loss. Maxilla is more commonly involved than mandible. When maxilla is affected it may involve zygomatic \& sphenoid bone. The pattern of which bones are involved is established very early in life and does not change with age. ${ }^{(5,6)}$

The plain film radiological features of FD are non-specific and vary widely. The normal bone is replaced by tissue that is more radiolucent, with a grayish "ground-glass" pattern that is similar to the density of cancellous bone but is homogeneous, with no visible trabecular pattern. Fries, has described three radiological patterns in craniofacial fibrous dysplasia. The first is pagetoid with bone expansion and alternate areas of radiopaque and radiolucency. It occurs in more than half of the patients, most of who are older than 30 years of age and have had symptoms for an average of 15 years. The second pattern is sclerotic, with bone expansion and a homogenous radio density (ground glass appearance). The third type is cyst-like, usually a round or oval lesion with a sclerotic border, which occurred in younger individuals. The incidence of pagetoidis type 56\%, sclerotic type $23 \%$, and the radiolucent type $21 \%$. The most effective method of monitoring growth and estimating extent of disease seems to be computerized tomography. ${ }^{(7,8)}$

The decalcified, H\&E stained sections show densely packed irregular shaped trabeculae of lamellar bone, showing lacunae and osteocytes, collagen matrix stroma with fibroblasts in an entangled standard with osseous trabeculate similar to the "Chinese writing", with no osteoblastic rimming. We described above includes a symptomatic and asymptomatic cases. The differential diagnosis includes Odontogenic cysts, Central ossifying fibroma, Aneurysmal bone cyst, Sclerosing Osteomyelitis, paget's disease. ${ }^{(9)}$ 
Although there was no compelling indication to seek a biopsy, any sudden change in the clinical presentation or behavior of the lesion might warrant further investigation. Malignant transformation of fibrous dysplasia ranges from $0.4 \%$ to $4 \%$. The recurrence rate of fibrous dysplasia falls within the range of 0 to $13.3 \%$. ${ }^{(10)}$ The main aim of the treatment is correction of the functionality in combination with aesthetic effects. The conservative therapeutical approach with limited reduction in the size of these lesions is enough to manage the symptoms. The radiotherapy increased malignant transformations more than 400 times. ${ }^{(11)}$

\section{Conclusion:-}

Fibrous Dysplasia is a benign disease that has the potential to cause significant cosmetic and functional disturbance. Much progress has been made over the past decade, for example the identification of the genetic mutation linked to the etiology of the disease. With proper understanding, diagnosis and management, good outcomes can often be achieved. Awareness of the myriad of radiographic appearances of fibrous dysplasia is essential and will be beneficial in the accurate diagnosis and proper treatment planning, even without invasive diagnostic procedures.

\section{References:-}

1. Shreedhar B, Kamboj M, Kumar N, Khan SS. Fibrous Dysplasia of the Palate: Report of a Case and Review of Palatal Swellings. Case Rep Pediatr 2012:1-4.

2. Chen YR, Chang CN, Tan YC. Craniofacial Fibrous Dysplasia: An Update. Chang Gung Med J 2006;29:543-9.

3. Abdelkarim A, Green R, Starzell J, Preece J. Craniofacial polyostotic fibrous dysplasia: a case report and review of the literature. Oral Surg Oral Med Oral Pathol Oral Radiol Endod. 2008;106:e49-e55.

4. DiCaprio MR, Enneking WF. Fibrous dysplasia. Pathophysiology, evaluation, and treatment. J Bone Joint Surg Am 2005;8;1848-64.

5. Farzaneh AH, Pardis PM. Central giant cell granuloma and Fibrous Dysplasia occurring in the same jaw. Med Oral Patol Oral Cir Bucal 2005;10:130-2.

6. Lee JS et al.Normal vision despite narrowing of the optic canalin fibrous dysplasia. N Engl Med J 2002;347:1670-6.

7. Nityasri V, Haris PS, Bose T, Balan A. Fibrous Dysplasia-A 13-year retrospective radiographic analysis in a south Indian population, Dento maxillofacial Radiol 2011;40:282-9.

8. Chen YR, Wong FH, Hsueh C, Lo LJ.Computed Tomography Characteristics of Non-Syndromic Craniofacial Fibrous Dysplasia. Chang Gung Med J 2002;25:1-8.

9. Buyuklu F, Tarhan E, Cakmak O, Ozgirgin N, Arikan U. Isolated fibrous dysplasia of the sphenoid sinus. Rhinology 2005;43:309-12.

10. MacDonald-Jankowski D. Fibrous dysplasia: A Systematic review. Dentomaxillofac Radiol.2009;38:196-215.

11. Parekh SF, Donthineni-Rao R, Ricchetti E, Lackman RD. Fibrous Dysplasia. Journal of the American Academy of Orthopedic Surgeons. 2004;12:305-13. 